\title{
Article \\ Experimental Implementation Of a Low Cost Full Analog Self-Jammer Canceller for UHF RFID Devices
}

\author{
Massimiliano Rossi ${ }^{1, t, \ddagger\left(\mathbb{D}, \text { Riccardo Maria Liberati }{ }^{1,+, \ddagger}, \text { Marco Frasca }\right.}{ }^{1,+, \ddagger(\mathbb{D}), J o h n}$ Richardson, ${ }^{3, \ddagger},{ }^{*}$ \\ MBDA Italia S.p.A. Via Monte Flavio, 45 - 00131- Roma - Italy - \\ X-Microwave LLC - 14050 Summit Drive - Suite 113B - Austin, TX 78728 \\ 1 massimiliano.rossi@mbda.it \\ 2 riccardo.liberati@mbda.it \\ 2 marco.frasca@mbda.it \\ 3 john.richardson@xmicrowave.com \\ * Correspondence: massimiliano.rossi@mbda.it; Tel.: +39-06-87711 \\ $\ddagger \quad$ These authors contributed equally to this work.
}

\begin{abstract}
The paper investigates the performance of a full analog self-jammer canceller able to operate in UHF RFID devices. The scheme has been realized using only SMT devices avoiding custom designed components. The paper analyzes the theoretical performance and experimental validation using a modular microwave technology approach.
\end{abstract}

Keywords: RFID; self-jammer; canceller

\section{Introduction}

Radio-frequency identification (RFID) is a widespread technology used to identify and track objects where small and usually passive tags are installed. Passive and batteryless tags are activated by a continuous wave $(\mathrm{CW})$ radiated by a reader and respond generating an iso-frequency backscattered field. These operations are simultaneous as the CW signal is requested to energize the tag while it answers to the reader, but the powers of the signals generated and received by the reader are quite different by several order of magnitudes. There are few ways to conveniently separate the transmitter and receiver paths as there are no separated frequency bands for the RF link. Tx and Rx paths can share a single antenna using a circulator or a directional coupler or two separated antennas can be used for receiving and transmitting. Dealing with real components, the actual separation can be too low as the transmitted and received signals are several orders of magnitude different and the CW signal generated by the transmitter can easily jam the receiver. This imposes heavy constraints on the low-noise amplifier stage in terms of dynamic range to avoid its saturation and the desensitization of the receiving channel. Several techniques have been proposed to mitigate this phenomenon that are not limited to the RFID (Radio-frequency Identification) system, where the use of a back-scatter communication exaggerates the effects, and several patents have been issued, for example [1] [2] .

\subsection{Discussion of related work and contribution}

Different approaches have been followed to try to improve the isolation between the transmitter and the receiver sections in RFID systems [3] [4], but some key points are very different from other applications. As the RF link is full-duplex, modern RF switches are not usable thus renouncing to a very high isolation degree, but fortunately, as communications are over short or very short range, the receiver can tolerate a high noise figure (up to several tens of $\mathrm{dB}[5]$ ). The solutions proposed in literature can be divided into:

1. analog approaches with the need for custom design of some key elements [6] [7] [8][9]

2. digital approaches with the need for custom design of active cancellation systems of different complexities [10] or recurring to custom integrated designs as [11] 
The purpose of this work is to propose a novel full-analog self-jamming canceller scheme and verify its feasibility using off-the-shelf components and an innovative modular microwave technology, without resorting to custom design.

\section{Materials and Methods}

A generic analog self-jam canceller is reported in Fig. 1 where a transmitter and a receiver share the same input/output port using hybrid couplers as isolating combiners. If all the devices are ideal, the TX/RX isolation is theoretically infinite, if a transmitter injects a signal $S_{\text {in }}$ into the circuit at port 1 , the corresponding output signal can be written as:

$$
S_{\text {out }}=S_{\text {in }} S_{31} S_{21 c} S_{41}+S_{i n} S_{41} S_{21 c} S_{42}=S_{\text {in }} S_{21 c}\left(S_{31} S_{41}+S_{i n} S_{41} S_{42}\right)=-j S_{\text {in }} S_{21 c}
$$

where $S_{21 c}$ is the corresponding scattering parameter of the circulators, supposed to be equals, and $S_{31}, S_{41}, S_{42}$ are the corresponding scattering parameters of the hybrid couplers.

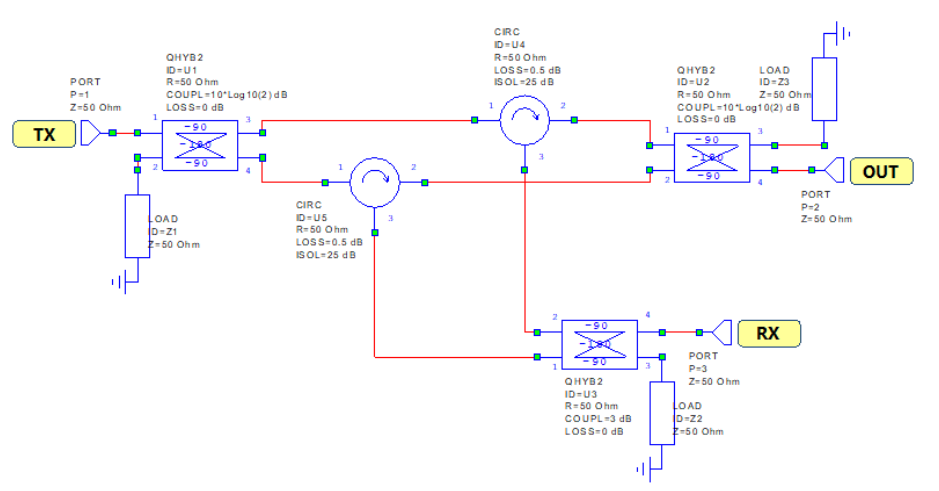

Figure 1. Generic Anti Self-Jam

A signal from port 2, $S_{r}$, is transferred to port 3 (receiver port) as:

$$
S_{r e c}=S_{r} S_{14} S_{21 c} S_{42}+S_{r} S_{24} S_{21 c} S_{41}=S_{r} S_{21 c}\left(S_{14} S_{42}+S_{24} S_{41}\right)=-j S_{r} S_{21 c}
$$

The theoretical signal arising from port 1 to port 3 is:

$$
S_{i s o}=S_{i n} S_{31} S_{31 c} S_{42}+S_{i n} S_{41} S_{31 c} S_{41}=S_{i n} S_{31 c}\left(\frac{1}{2}-\frac{1}{2}\right)=0
$$

results indicate a nearly perfect isolation and a very low loss path from port 1 to port 2 , as indicated in Fig. 2

Some strong assumptions have been made in the previous analysis, the two circulators are assumed to be identical and the same is true for the three hybrid couplers. Moreover, not only the couplers have been considered equal, but perfectly matched and with an infinite isolation between ports 1,2 and 3,4. Using actual devices the situation changes deeply. A finite isolation between the input ports of the hybrid couplers, usually worse than the reverse isolation of a generic circulator operating in the same band, creates two spurious paths that make the isolation between the transmitter and receiver ports of the circuit vanish. In fact, neglecting relections, it is possible to write:

$$
S_{\text {iso }}=S_{i n} S_{31} S_{31 c} S_{42}+S_{i n} S_{41} S_{31 c} S_{41}+S_{i n} S_{31} S_{21 c}^{2} S_{21} S_{41}+S_{i n} S_{41} S_{21 c}^{2} S_{12} S_{42}
$$

thus, the isolation can be put as:

$$
S_{i s o}=S_{i n} S_{31 c}\left(S_{31} S_{42}+S 41^{2}\right)+S_{i n} S_{41} S_{21 c}^{2}\left(S_{31} S_{21}+S_{12} S_{42}\right)
$$




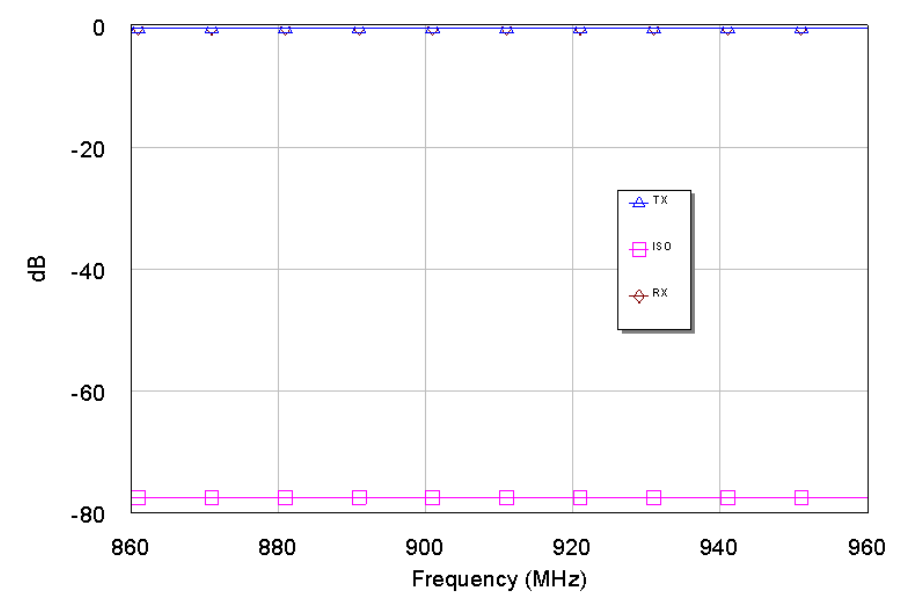

Figure 2. Generic anti self-jam performance

so

$$
S_{i s o}=S_{i n} S_{31 c}\left(S_{31} S_{42}+S 41^{2}\right)+S_{i n} S_{41} S_{21 c}^{2}\left(S_{31} S_{21}+S_{12} S_{42}\right) \neq 0
$$

as and hybrid coupler is a passive and reciprocal device, $S_{21}=S_{12}$, Eq. 6 can be rewritten as:

$$
S_{i s o}=S_{i n} S_{31 c}\left(S_{31} S_{42}+S 41^{2}\right)+S_{i n} S_{41} S_{21 c}^{2} S_{21}\left(S_{31}+S_{42}\right) \neq 0
$$

If the isolation of the circulators can be assumed to be high enough, the dominant contribution to $S_{i s o}$ is due to the isolation of the hybrid coupler connected to port 2 of the schematic. It is to be remarked however that eq. 7 does not take into account reflections that still exist. Replacing the ideal hybrid couplers with actual devices, specifically Mini-Circuits $Q C N-12 A$, the performance degrades, as reported in Fig.3 the cancellation is quite similar to the isolation parameter of the hybrid coupler

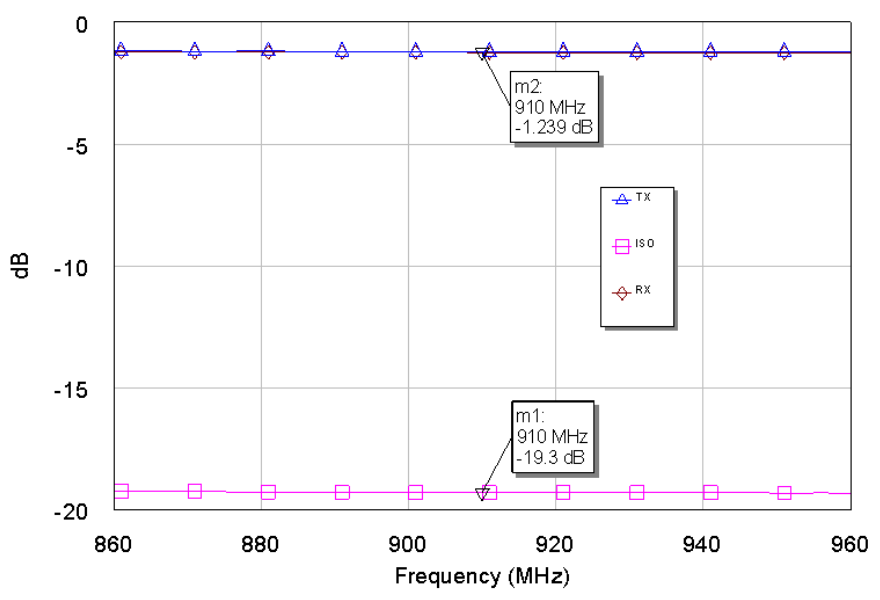

Figure 3. Generic anti self-jam performance with actual hybrid couplers in place

reported in Fig.4 


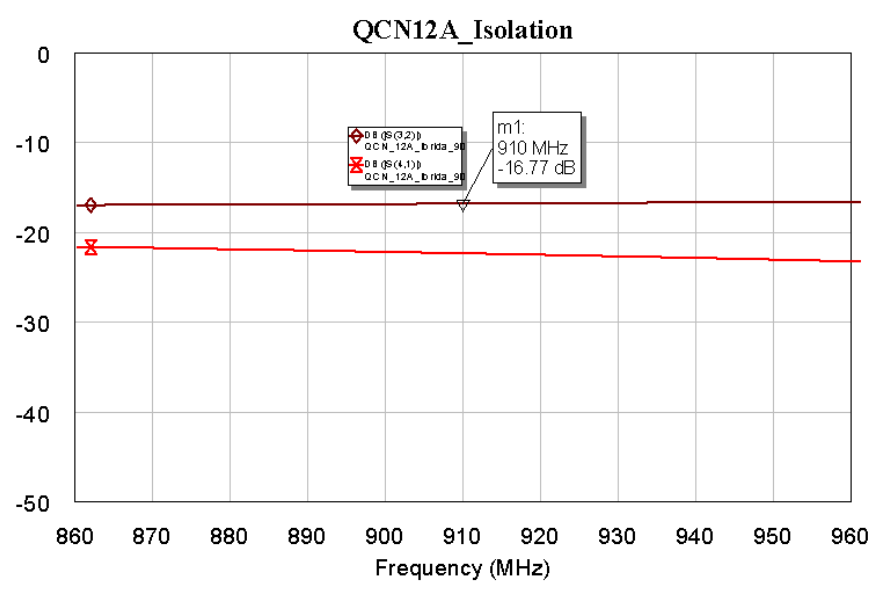

Figure 4. Isolation performance of QCN12A Hybrid Coupler

\subsection{Proposed solution}

The topology of Fig.1 can be conveniently modified in order to try to overcome the intrinsic limitation due to the nature of the actual devices. Looking at Figure 5, the transmitted signal injected into port 1 is split in two separated and specular branches out of phase. The first, the upper, sees the signal travelling through a circulator and toward port 3 of an hybrid combiner. The signal coming from the lower branch terminates into port 2 of the same hybrid combiner. The signals, that are 180 degrees out of phase are combined by an additional 90 degree phase shift. These signals are ready to be used for powering, as an example, a sequentially rotated patch array in order to generate a circular polarized beam without the need for an external 180 degree hybrid. The signal injected in port 1 that is split in two anti-phase branches reaches ports 3 of the circulators and is summed. The spurious paths from port 1 of the circuit to port 2 and 3 of the zero degree power combiner, passing through port 3 and 2 of the 90 degree output hybrid combiner cancel each other at port 3 (RX) of the circuit. Signals coming from port 2 and 4 of the circuit are combined through the hybrid combiner and summed at port 3 of the circuit.

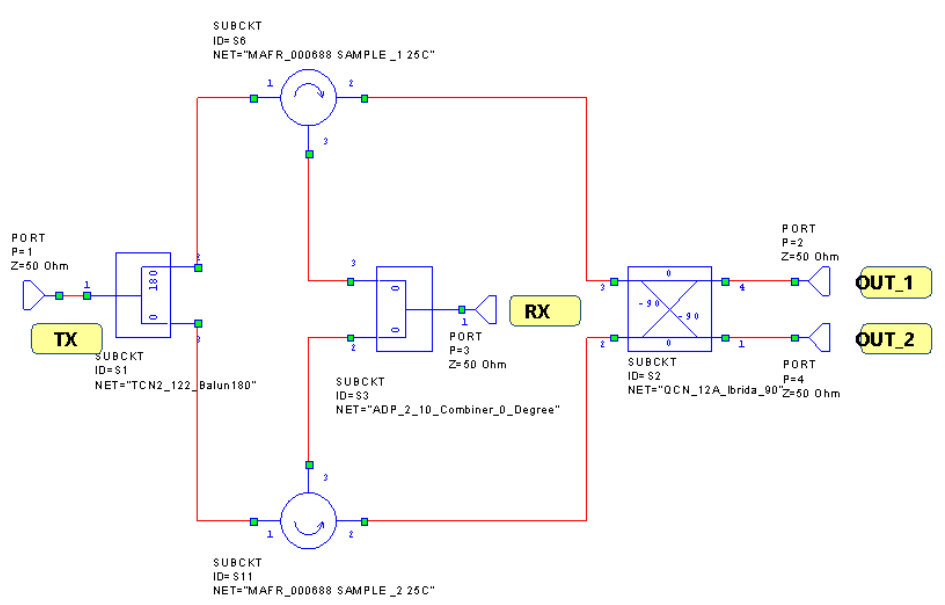

Figure 5. Proposed solution

The circuit can be analyzed in detail splitting it in three main parts: the input network made by the 180 degree splitter and the circulators, the 90 degree hybrid combiner and the zero degree combiner 
on the receiving path. With reference to Fig. 6 green, red and dashed red arrows represent respectively direct, undesired and reflected signals. Considering the hybrid combiner terminated in matched loads, the transmitted wave $b_{3}$ on the upper path can be written as:

$$
b_{3} \approx S_{41} S_{67} S_{32}+S_{21} S_{66} S_{32}+S_{31}
$$

while the transmitted wave $b_{5}$ on the lower path can be written as:

$$
b_{5} \approx S_{21} S_{76} S_{54}+S_{41} S_{77} S_{54}+S_{51}
$$

the output signal, that is the isolation contribution, $S_{i s o}$ can be described as:

$$
S_{\text {iso }} \approx S_{1311} b_{3}+S_{1312} b_{5}
$$

some considerations can be made: the hybrid combiner is a passive and reciprocal network,so

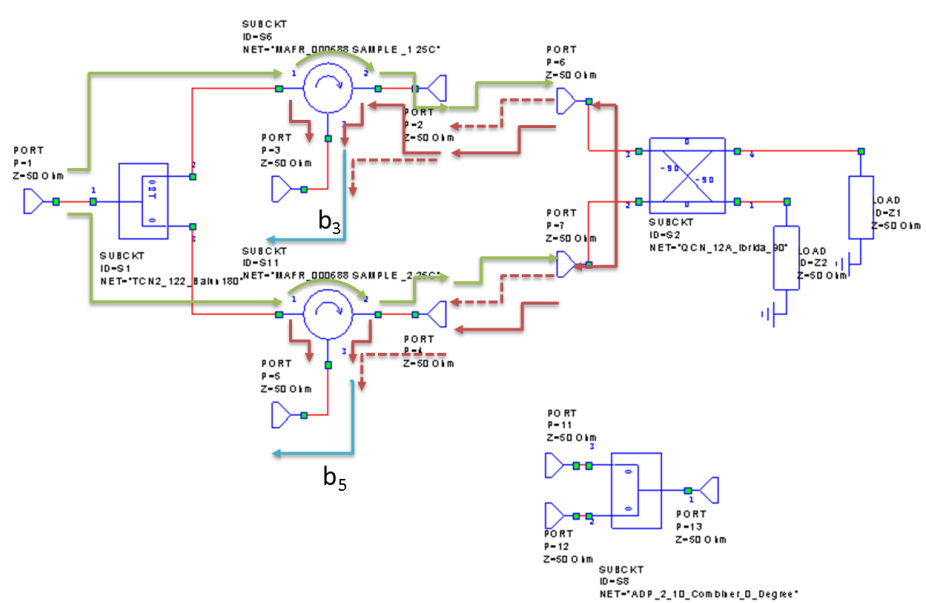

Figure 6. Circuit separation for analysis

$S_{67}=S_{76}$ while it can be assumed that $S_{32} \approx S_{54}=S_{o}$ and $S_{21} \approx-S_{41}=S_{d}$, with $S_{o}$ and $S_{d}$ as the scattering parameters of the forward path of the circulators (from port 2 to port 3 of the devices) and the path from port 1 to port 2 of the circuit respectively. Eq. 8 can be written as:

$$
b_{3}=S_{d} S_{o}\left(S_{67}-S_{66}\right)+S_{31}
$$

and Eq.9 as:

$$
b_{5}=S_{o} S_{d}\left(-S_{76}+S_{77}\right)+S_{51}
$$

if $S_{1311} \approx S_{1312}=S_{\text {sum }}$ than:

$$
b_{5}=S_{o} S_{d}\left(-S_{76}+S_{77}\right)+S_{51}
$$

for the zero degree combiner it can be assumed that $S_{1311} \approx S_{1312}=S_{\text {sum }}$ so that the isolation can be written as:

$$
S_{i s o}=S_{d} S_{o} S_{\text {sum }}\left(S_{67}-S_{66}-S_{67}+S_{77}+S_{51}+S_{31}\right)=S_{d} S_{o} S_{\text {sum }}\left(-S_{66}+S_{77}\right)+S_{d} S_{o} S_{\text {sum }}\left(S_{51}+S_{31}\right)
$$

Eq.14 suggests that if $S_{66}=S_{77}$ and $S_{51}=-S_{31}$ then the isolation could be infinite, but there are also other favorable combinations as in actual devices the $S_{x x}$ parameters can be of the same order of magnitude of circulators isolation obtaining $\left|S_{d} S_{o} S_{\text {sum }}\left(-S_{66}+S_{77}\right)\right| \approx\left|S_{d} S_{o} S_{\text {sum }}\left(S_{51}+S_{31}\right)\right|$. Isolation is theoretically granted even if ports 1 and 4 of the hybrid coupler are closed with equal but 
mismatched loads. In this case the contribution due to the reflected waves from the transmitter are summed in out of phase at the receiver power combiner.

\section{Simulated and Experimental Results}

The circuit has been simulated with NI-AWR Microwave Office [12] using the measured and available scattering parameters of the selected devices that are all SMT, low cost cots units:

- $\quad$ Mini-Circuits TCN2 - 122 Balun

- $\quad$ Skyworks MAFR000688 Circulators

- Mini-Circuits ADP-2-10 in-phase Power Combiner

- Mini-Circuits QCN-12A 90 degree Hybrid Combiner

The simulated performance of the circuit is reported in Fig. 7 where the isolation from transmitting and receiving ports is well over $30 \mathrm{~dB}$ from 860 to $960 \mathrm{MHz}$. The insertion losses from ports 2 and 4 to port 3, the receiver, is quite similar to the insertion loss from port 1 to ports 2 and 4 and around $4.5 \mathrm{~dB}$. Moreover, results predicted by Eq.10 are reported in Fig. 8 together with those coming from

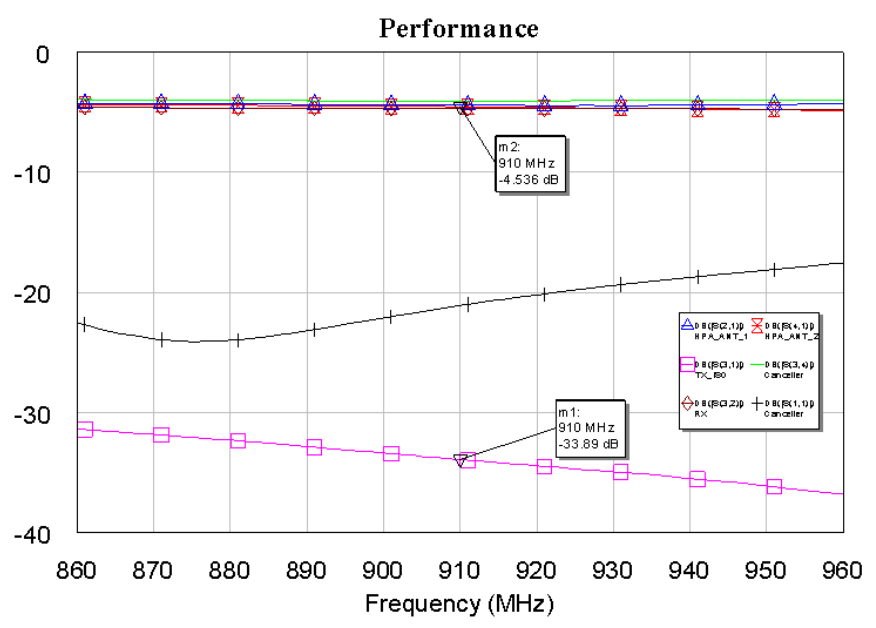

Figure 7. Simulated performance

the simulation of all the circuit and are very close each other within an error of $0.2 \mathrm{~dB}$. The actual carrier over interference ratio at the receiver port is reported in Fig. 9 where it is clearly visible an average isolation of about $35 \mathrm{~dB}$ all over the bandwidth. The scheme has been realized using an innovative rapid prototyping and modular technique by X-Microwave [13]. The circuit has been sectioned into smaller drop-in parts called X-MWblocks and each one is devoted to a specific task as clearly visible in Fig. 10. The proposed technique allows rapid and reliable prototyping and the opportunity to tune the hardware without having to redesign each time. Each drop-in has been realized with a 49 mil 4-layer stackup PCB using 8 mil Rogers RO4003 substrate as top Core. The input port of the circuit, port 1, is probed with a high performance $2.92 \mathrm{~mm}$ connectorized microwave probe (X-MWprobe). The RF probe is connected to a dedicated 0409 format pcb (i.e. $4 \times 9$ grid spaces), using CPWG (Coplanar Waveguide with Lower Ground Plane) microstrip transmission, populated with a Mini-Circuits TCN2 - 122 Balun. The Skyworks MAFR000688 circulators have been integrated on two 1009 format pcb's and the connections with the TCN2 - 122 have been granted by two 50 Ohm "S" shaped transmission lines. Ports 2 of the circulators have been connected to the cascade of a couple of small transmission lines having the main function to realize the RF paths, but to be easily replaced with other components, if necessary. Two auxiliary 0409 format "S" shaped transmission lines close the electrical paths from the transmitter port to the QCN-12A 90 degree hybrid combiner 


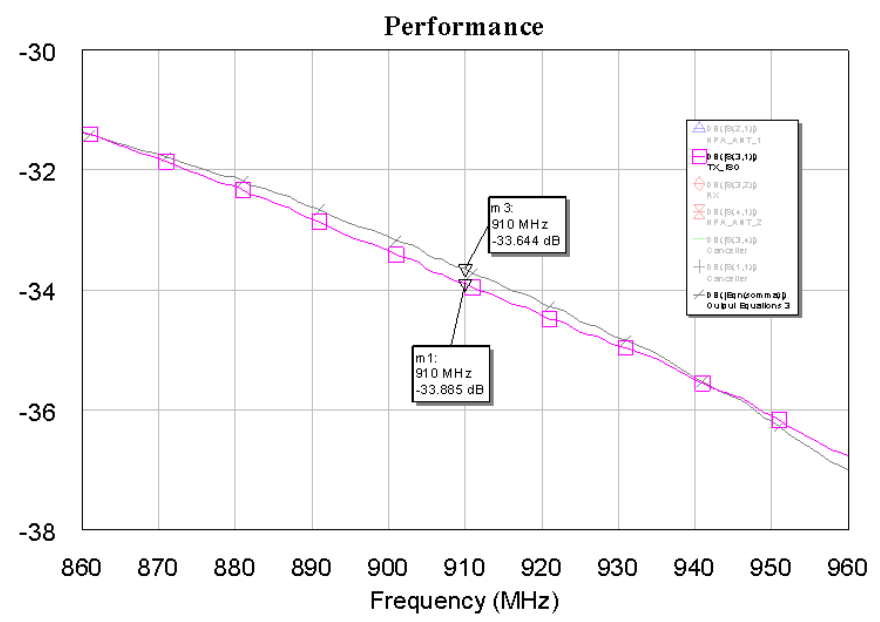

Figure 8. Simulated isolation performance and theoretical results comparison

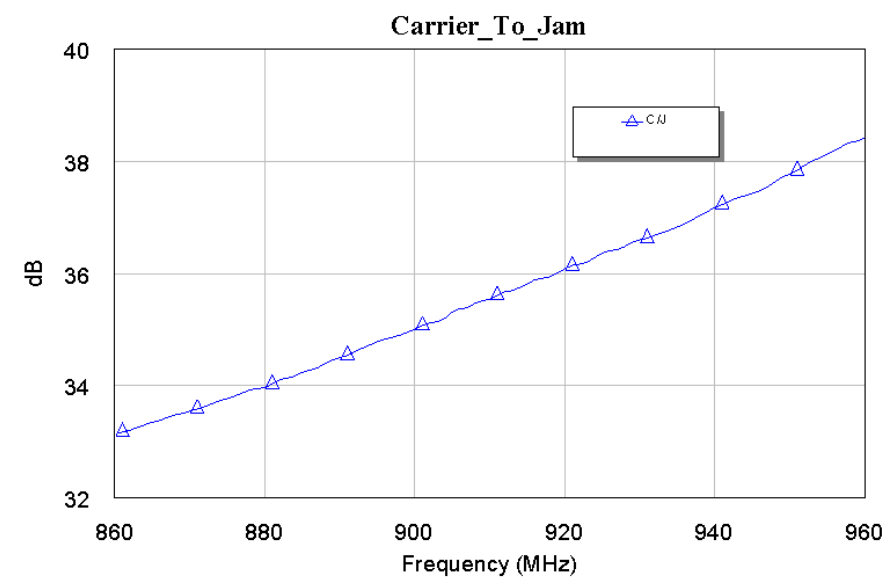

Figure 9. Simulated carrier over interference ratio

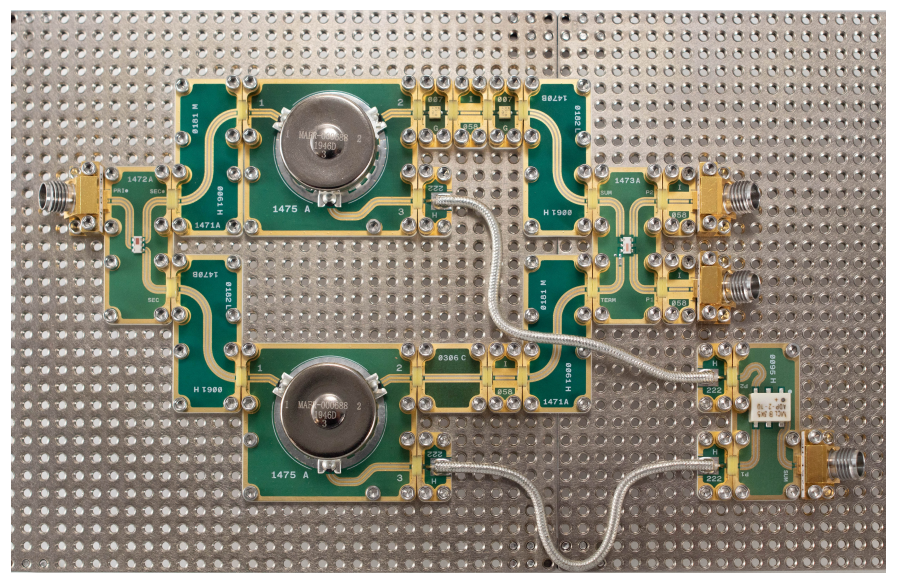

Figure 10. Overview of the circuit from above

integrated on a dedicated 0409 format board. The output ports of the hybrid combiner are connected to two high performance $2.92 \mathrm{~mm}$ probes. Ports 3 of the two circulators are connected to the pcb 
with the Mini-Circuits ADP-2-10 in-phase power combiner on board through two phase-matched hand formable coaxial cables. All the interconnections between the boards are performed by a special ground-signal-ground jumpers (gsg jumpers) that are small pieces of polyimide material with copper traces that are shaped to mantain $50 \mathrm{ohm}$ impedance while bridging the 5 mil air gap from launch to launch of adjacent boards. The copper traces are coated with diamond particles and then gold-plated creating diamond particle interconnect. The gsg jumpers are then laid across each port of two boards and two anchors are used to hold them firmly against the PCB on each side of the connection as clearly visible in Figure 11. Every microstrip PCB is screwed to a metal prototype plate using dedicated screws

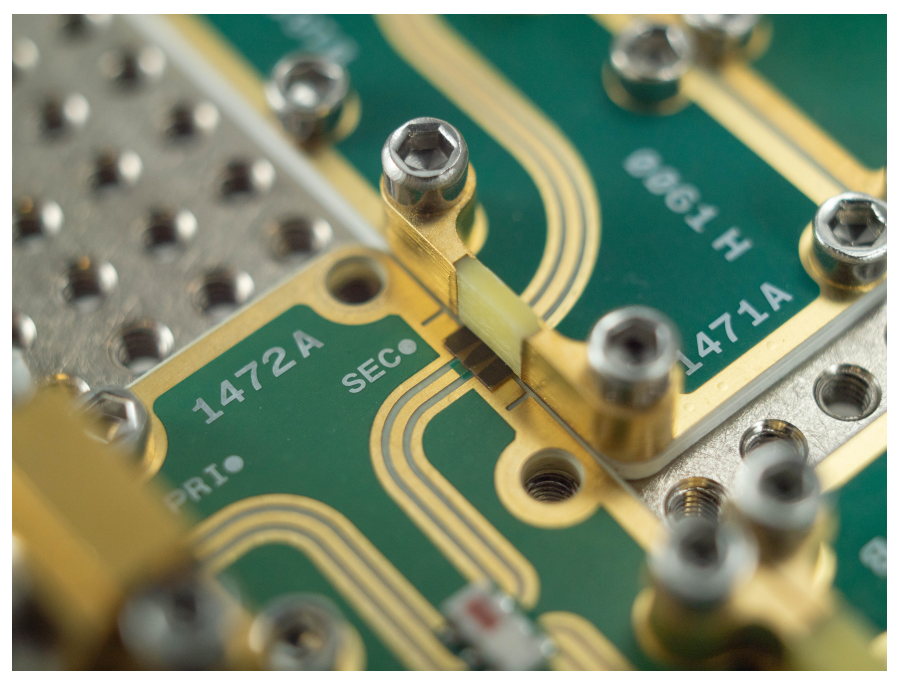

Figure 11. Detail of PCB interconnection with one jsg jumper removed

in order to obtain a mechanically robust assembly while assuring a very good ground path to all the devices. The circuit has been characterized using a Keysight PNA-X N5247B 67 GHz Vector Network Analyzer [14] extracting the 4-port matrix of the circuit as visible in Figure 12. The simulated and measured input matching of the 4-port circuit are reported in Figure 13 


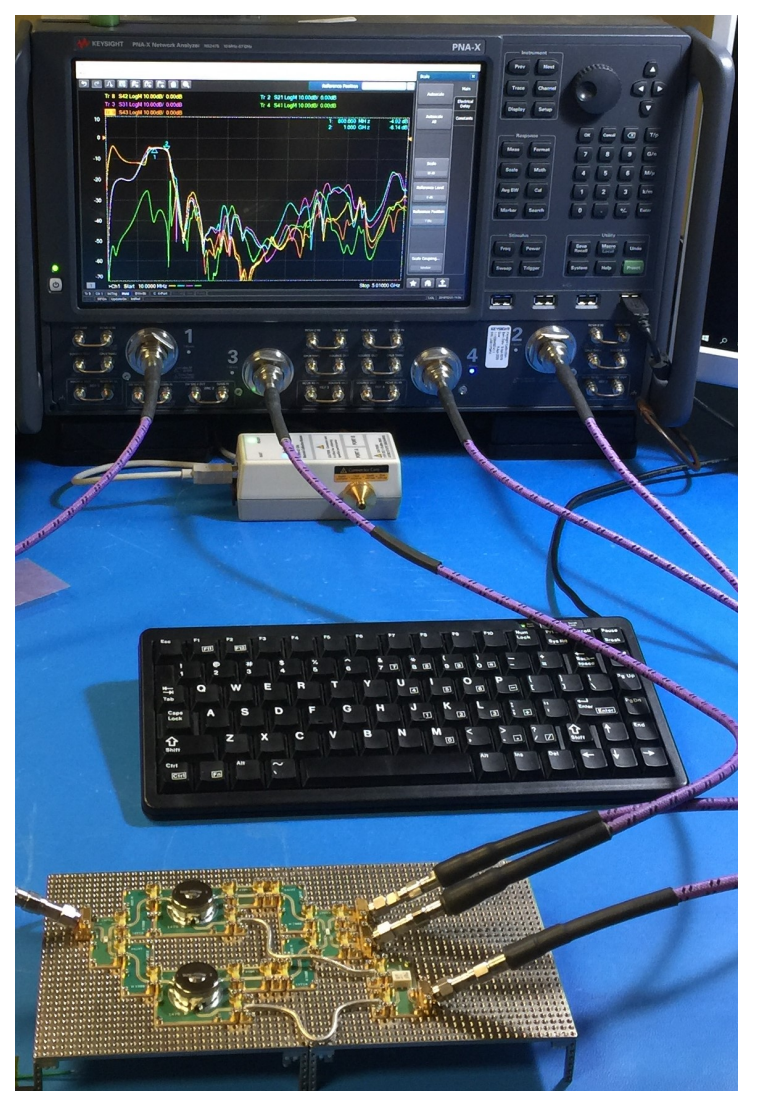

Figure 12. Characterization set-up with the VNA in place

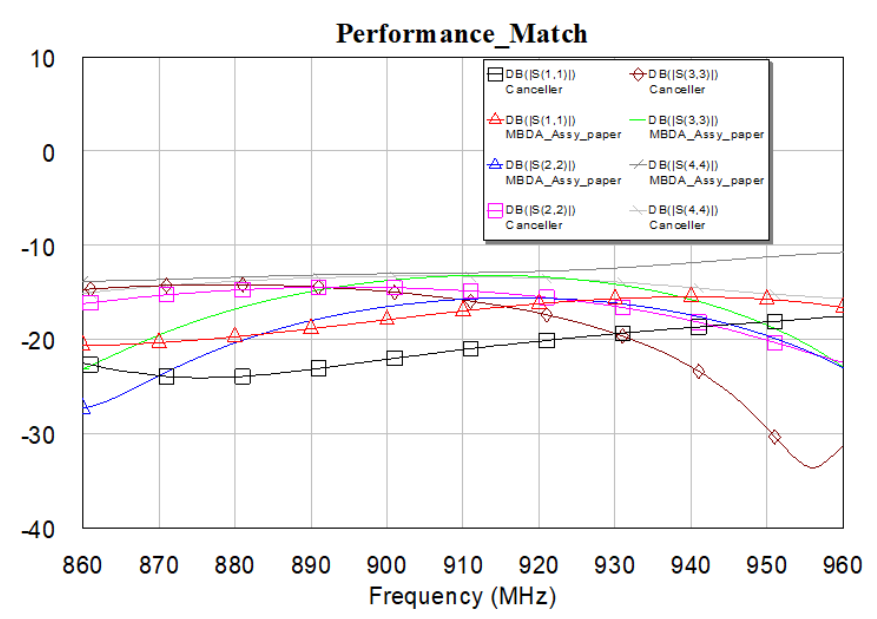

Figure 13. Experimental and simulated input matching

while the simulated and measured insertion losses of the transmitting paths are reported in 14 where the difference between the theoretical and actual values are within $1 \mathrm{~dB}$. The isolation between the input port (transmission) and the receiver port is visible in Figure 15. The experimental data concerning the isolation parameter are slightly better than the simulated by $\sim 1.5 \mathrm{~dB}$ 


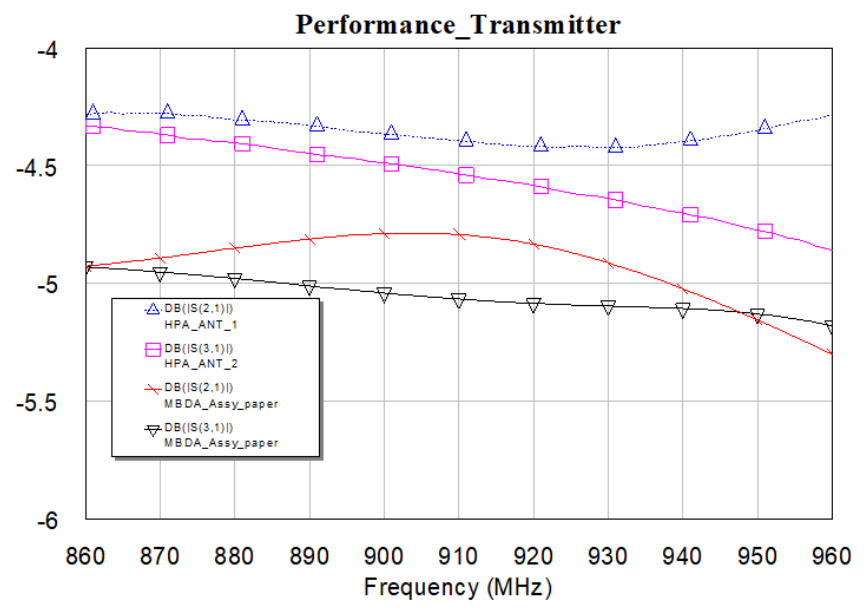

Figure 14. Experimental and simulated insertion losses of the transmitting path

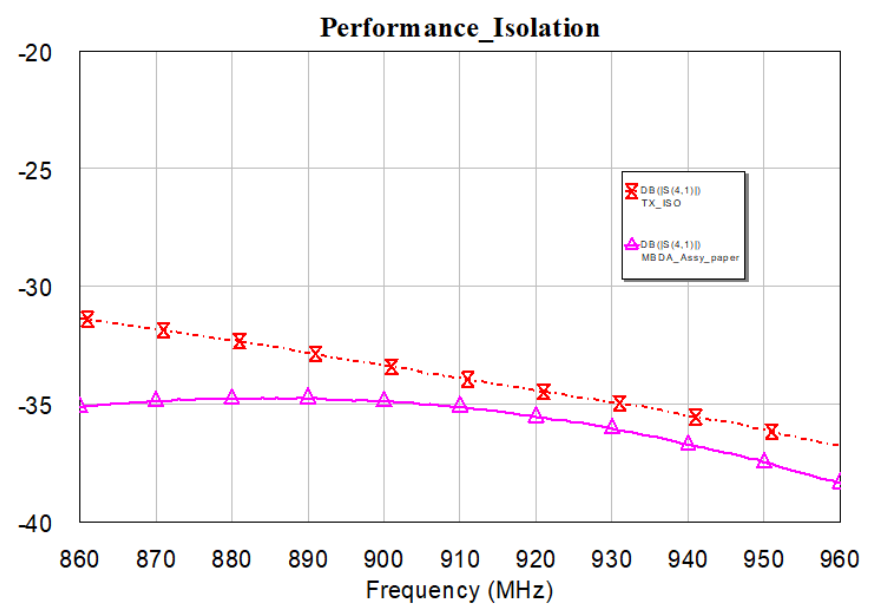

Figure 15. Simulated isolation and experimental result comparison

The carrier over jammer suppression performance of the scheme is visible in Figure 16 where the actual performance is slightly better than the simulated within 0.5 to $3 \mathrm{~dB}$ over the entire bandwidth. 


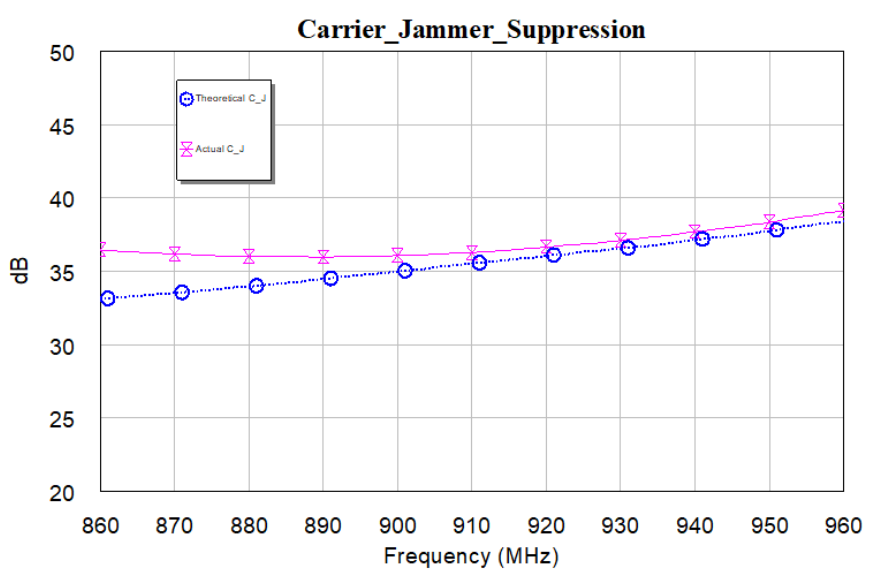

Figure 16. Experimental and simulated carrier over jammer suppression

\section{Discussion}

In this paper a novel low-cost full analog anti self-jam circuit suitable for UHF RFID has been presented. The theory has been developed with the exclusive use of low-cost components in SMT technology without requiring specific or custom design. The validation has been carried out through the innovative modular technology made available by X-Microwave through which it was possible to create a prototype and verify its performance in a very short time obtaining even better results than those expected from the simulation. The next step is to optimize the circuit, having surely margins available, in order to reach a compact and performing circuit.

Author Contributions: All authors contributed to this paper. Massimiliano Rossi proposed the main idea, working on system design, devices selection and simulations, data analysis and paper editing. John Richardson contributed to the paper editing performing all the hardware design, assembly and experimental characterizations. Marco Frasca and Riccardo Liberati contributed to the theoretical analysis and critical review of the work. All authors have read and agreed to the published version of the manuscript

Funding: This research received no external funding

Conflicts of Interest: The authors declare no conflict of interest.

\section{Abbreviations}

The following abbreviations are used in this manuscript:

CPWG Coplanar Waveguide with Lower Ground Plane

$\mathrm{CW} \quad$ Continuous Wave

PCB Printed Circuit Board

UHF Ultra High Frequency

RFID Radio-frequency Identification

SMT Surface Mounting Technology

\section{References}

1. Thinh Q. HoStephen, M. HartGregory, A. Kosinovsky Willard, I. Henry, US Patent 5815803A, March 1996

2. Siu K. Cheung, Willam H. Weedon III, Timothy Philip Halloran, US Patent 7541890B2, June 2009

3. A. Ghahremani, V. D. Rezaei and M. Sharif Bakhtiar, "A UHF-RFID Transceiver With a Blocker-Canceller Feedback and +30 dBm Output Power," in IEEE Transactions on Circuits and Systems I: Regular Papers, vol. 60, no. 11, pp. 3043-3054, Nov. 2013. doi: 10.1109/TCSI.2013.2252671 
4. Boaventura, A.J., Santos, J., Oliveira, A.S., Carvalho, N.B. (2016). Perfect Isolation: Dealing with Self-Jamming in Passive RFID Systems. IEEE Microwave Magazine, 17, 20-39.

5. Usachev, Nikolay Elesin, Vadim Nikiforov, A. Chukov, George Nazarova, Galina Sotskov, Denis Shelepin, Nikolay Dmitriev, Vladislav. (2015). System design considerations of universal UHF RFID reader transceiver ICS. Facta universitatis - series: Electronics and Energetics. 28. 297-307. 10.2298/FUEE1502297U.

6. J. Chen, T. Yan, Z. Huang, J. Li and G. Wen, "Carrier and Noise Cancellation Effects in a Self-Jammer Canceller for UHF RFID Readers," 2018 International Conference on Microwave and Millimeter Wave Technology (ICMMT), Chengdu, 2018, pp. 1-3. doi: 10.1109/ICMMT.2018.8563848

7. Desong Wan, Bin You and Lingling Sun, "Radio link analysis and design of directional coupler with high isolation for UHF RFID reader," 2008 11th IEEE International Conference on Communication Technology, Hangzhou, 2008, pp. 256-259. doi: 10.1109/ICCT.2008.4716225

8. W. Kim et al., "A Passive Circulator with High Isolation using a Directional Coupler for RFID," 2006 IEEE MTT-S International Microwave Symposium Digest, San Francisco, CA, 2006, pp. 1177-1180. doi: 10.1109/MWSYM.2006.249401

9. A. Sadeghfam and H. Heuermann, "Electrically Tunable Bandpass Filter with Integrated Carrier Suppression for UHF RFID Systems," 2008 38th European Microwave Conference, Amsterdam, 2008, pp. 1727-1730. doi: 10.1109/EUMC.2008.4751809

10. W. Kim et al., "A Passive Circulator with High Isolation using a Directional Coupler for RFID," 2006 IEEE MTT-S International Microwave Symposium Digest, San Francisco, CA, 2006, pp. 1177-1180. doi: 10.1109/MWSYM.2006.249401

11. Q. Guo, Y. Zhai, X. Tan and H. Min, "An On-Chip Configurable Receiver With $>55-\mathrm{dB}$ Tx Leakage Suppression for UHF RFID Reader," in IEEE Microwave and Wireless Components Letters, vol. 29, no. 5, pp. 357-359, May 2019. doi: 10.1109/LMWC.2019.2907173

12. AWR Microwave Office, https://www.awr.com/

13. X-Microwave, https://www.xmicrowave.com/

14. Keysight N5247B VNA, https://www.keysight.com/en/pdx-3025561-pn-N5247BM/active-devicecharacterization-solution-up-to-67-ghz?nid=-32497.1277564\&cc=US\&lc=eng 